\title{
Influence of Fibers on the Dust Dislodgement Efficiency of Bag Filters
}

\author{
L. Bao, ${ }^{1}$ M. Musadiq, ${ }^{1}$ T. Kijima, ${ }^{2}$ and K. Kenmochi ${ }^{1}$
}

*1 Faculty of Textile Science and Technology, Shinshu University, 3-15-1 Tokida Ueda-shi, Nagano-ken 386-8567 Japan

*2 Nihon Spindle Manufacturing Co., Ltd., 4-2-30 Shioe, Amagasaki-city, Hyogo, 661-8510, Japan

\begin{abstract}
In recent years, non-woven bag filters have been used in waste incinerators for efficient collection of dust and removal of detrimental gas. However, dust dislodgement efficiency decreases with time until the bag filters are no longer useable. Dust adhering to the fabric is a major determinant of bag filter life. In this study, a flat filter was used to study the relationship between various parameters of a bag filter's structure and dust dislodgement efficiency. The results confirm that fiber linear density, fiber modulus of elasticity, and forms of fiber cross-section of felt in bag filters affect the dust dislodgement efficiency of a bag filter.

Higher fiber linear density in a bag filter prevents dust from penetrating the filter, thus helping the filter to dislodge dust easily. Application of various forms of fiber cross-section indicated that with the same fiber linear density, the triangular form was better than a circular form. A lower Young's modulus allows the fiber to bend easily and prevents the dust from penetrating the bag filter. Fiber linear density, fiber modulus of elasticity, and fiber cross-section form are thus the elements that affect bag filter efficiency
\end{abstract} loss.

Key words: Bag filter, Fiber linear density, Fiber modulus of elasticity, Dust dislodgement efficiency, Residual pressure

\section{INTRODUCTION}

With the rapid development of industrial activities, harmful substances beyond the purification ability of nature have been discharged, and their influence on our living environment and the ecosystem has become a major problem. Therefore, various countermeasures have been adopted at industrial sites in an effort to prevent atmospheric pollution. Dust-collection technology to separate dust in the air flow from the air flow discharged from production equipment is one such countermeasure. Currently, bag-filter dust collectors are typically used in garbage incineration facilities and blast furnaces.

As illustrated in Fig. 1, a bag filter uses the outer surface of a filter (backing fabric lined with felt on both sides) as the filtering face, and dust gas is filtered through this surface. Dust is dislodged by jetting pulsated compressed air onto the inner side of the filter. Accumulated dust is peeled off using the power of compressed air and by deformation of the filter. Bag-filter dust collectors are currently the most widely used dust collectors because of their high dust-collecting efficiency and low investment cost.

Many studies have focused on the dust-collecting characteristics of the bag filter, and the Association of Powder Industry and Engineering, Japan ${ }^{1}$ has contributed greatly to the design and operation of dust collectors by summarizing these studies. However, the use of pulsed jet air to dislodge dust from the filter is essential for continuous operation of dust collectors. Improvement of dust dislodgement efficiency increases the service life of bag filters and decreases their running cost. Thus, both dust collection and dust dislodgement are important.

More studies have addressed dust collection than the factors that influence dust dislodgement efficiency. However, several researchers have reported on this topic. Hindy et al. ${ }^{2}$ investigated the relationship between the differential pressure with acceleration in the filter section and the dust dislodgement efficiency of a cylindrical bag filter. The weight per area of the filter influences dust dislodgement efficiency. Simon et al. ${ }^{3}$ investigated the influence of nozzle size and jetting position on the movement of a cylindrical bag filter. Dennis et al. ${ }^{4}$ studied the influence of the surface of the filter material on filter movement. According to this study, accumulated dust dislodgment efficiency is influenced by both the material and the filter surface treatment. Ellenbecker et al. ${ }^{5}$ conducted experiments using a flat filter and investigated the relationship between dust dislodgement efficiency 
and cloth acceleration. They demonstrated that dust dislodgement efficiency improves even when acceleration increases, and that efficiency rapidly improves with a large volume of accumulated dust. Dennis et al. ${ }^{6}$ conducted dust dislodgement experiments using collision on a filter where dust accumulated and found that greater volume of accumulated dust and collision acceleration yielded higher dust dislodgement efficiency. Furthermore, they reported that high acceleration must act continuously for dust dislodgement. The authors ${ }^{7}$ proposed a multi-pulse jet system in which pulse parameters alone are changed while the current dust dislodgement system is used as it is. Results indicated that the dust dislodgement efficiency of our proposed multi-pulse dust dislodgement system improved by ten percent, if the same air volume was consumed for dust dislodgement. However, few studies have focused on the influence of fiber diameter and weight per area in the structure of the bag filter on dust dislodgement efficiency. Therefore, many issues remain unclear.

In this study, we focused on the influence of the fiber structure of the felt bag filter (diameter, form, and Young's modulus of fiber) on dust dislodgement efficiency in an effort to improve the dust dislodgement efficiency of bag filters. We prepared bag filters with different parameters, conducted dust dislodgement experiments, examined the influence of fiber structure on dust dislodgement efficiency, and explored its mechanism.

\section{Experimental}

\section{Construction of Bag-Filter Samples}

To investigate the influence of fiber characteristics on the bag filter efficiency performance, six types of bag filter for dust collection in blast furnaces (Table 1) were provided by the Japan Spindle Co., Ltd., or manufactured as prototypes. The felt fiber is polyester for all types. Sample 1 is a commercially available reference product. Samples 1, 3, and 6 vary in fiber linear density $(2.2,1.7$, and 1.4dtex) but have the same values of other parameters. Samples 2, 3, and 5 vary in fiber strength (manufacturer's value) but have the same values of other parameters. Samples 1 and 4 vary in the cross-sectional form of fibers; that of Sample 4 is triangular. The thickness of the filter is $2 \mathrm{~mm}$, and the density of the bag filter is $300 \mathrm{~kg} / \mathrm{m}^{3} \pm 2$, and the porosity of filters is 0.782 .

\section{Description of Test Equipment}

The dust-collection equipment (Fig. 2) used in this study conforms to Japanese Industrial Standard (JIS) Z 8909-1. This equipment consists of a dust feeder, a dispersion unit, a bag filter, a discharge fan, a jet-pulse cleaning system, and a system for measuring pressure loss and flow flux.

The filter sample was fixed on a frame (13) and set between the clean side and the dust side. Dust was added to the dust supply vessel (8), and the filtration process began. The total filtration surface area was $0.314 \mathrm{~m}^{2}$. Filtration was conducted until the pressure loss reached $1000 \mathrm{~Pa}\left(\square \Delta P_{\text {control }}\right.$ ), at which time the dust dislodgement process began. After pulse injection, the pressure loss $\left(\Delta P_{\text {collect }}\right)$ of the filter was measured. The filtration experiment conditions are presented in Table 2, and the dust dislodgement experiment conditions are presented in Table 3.

Fig. 3 illustrates the relationship between pressure loss and time. Here, $\Delta P_{\text {initial }}$ is the pressure loss of a new filter medium. As filtration continues, pressure loss increases until it reaches the control pressure loss $\left(\Delta P_{\text {control }}\right)$ of $1000 \mathrm{~Pa}$; then the dust dislodgement process begins. After the dust is dislodged, the pressure loss decreases, and filtration starts again. When a new filter is applied, its initial pressure loss $\left(\Delta P_{\text {Initial }}\right)$ is low. The residual pressure loss $\left(\Delta P_{c i}\right)$ increases during dust collection due to the accumulation of undislodged dust. The difference between residual pressure loss and the control value of pressure loss $\left(\Delta P_{\text {Control }}\right)$ decreases, and dust must be dislodged frequently by jetting pulsated air, or power consumption increases and the service life of the filter decreases.

To ensure that a new filter is like a filter that has been used on an actual machine (dust penetrates the filter) within a short time, more dust was made to stick to the filter by the vibration and air flow proposed in our last report $^{7}$ using the sticking accelerating test equipment, which makes dust quickly penetrate the filter (Fig. 4). The pressure of the gas diffusion section is set to 500Pa. A mass of dust $(40 \mathrm{~g})$ is applied on the filter. With gas diffused, the filter is vibrated for $48 \mathrm{hrs}$ to accelerate the penetration of dust into the filter. 


\subsection{Experiment Description}

Tests were conducted in several stages. During the first stage, the dust dispersion vessel was disconnected, and the pressure loss of a new filter medium $\left(\Delta P_{\text {initial }}\right)$ was recorded for a flow of clean air. Injected compressed air was then discharged, and the responses of the various sensors were recorded. The purpose of this first series of experiments was to determine the reaction of a new filter medium to jet-pulse injection of compressed air.

The second stage involved loading the filter medium onto the shaking device. With gas diffused, the filter is vibrated for $48 \mathrm{~h}$ to accelerate the penetration of dust into the filter. After the filter medium was processed by the shaking device, the filtration and dust dislodgement processes were conducted for 10 cycles for each filter medium. The residual pressure loss $\left(\Delta P_{c i}\right)$ of the filter medium was recorded, and the dust dislodgement efficiency $(\eta)$ was determined by the equation below.

$$
\eta=\Delta P_{\text {initial }} / \Delta P_{c i}
$$

Six samples are used for each filter sample.

Mechanical properties of the filter's fiber sample were determined by Auto Graph (AG-20KND) with a load cell $(1 \mathrm{~N})$ manufactured by Shimadzu Corporation. The sample length is $100 \mathrm{~mm}$, and the test speed is $2 \mathrm{~mm} / \mathrm{min}$. The fiber Young's modulus is determined. Ten samples are used for each fiber sample.

\section{RESULTS AND DISCUSSION}

\section{Influence of Fiber Diameter}

The fiber aggregate in the bag filter greatly influences the dust collection of the dust collector: A smaller fiber diameter yields better dust-collection. ${ }^{1}$ Dust dislodgement efficiency was measured for Samples 1, 2, and 6 to investigate the influence of fiber diameter on the dust dislodgement efficiency of the bag filter.

The filter is made of polyester with a fiber density of $1.38 \times 10^{3} \mathrm{~kg} / \mathrm{m}^{3}$. The diameter of the fiber of Sample 1 is $14.2 \mu \mathrm{m}$; that of Sample 2 is $12.4 \mu \mathrm{m}$; and that of Sample 6 is $11.3 \mu \mathrm{m}$. Figure 5 compares the dust dislodgement efficiencies of samples with different fiber diameters. The horizontal axis represents the fiber diameter. Samples 2 and 6 have fibers that are thinner than that of Sample 1 (commercial product). The other parameters are about the same.

A greater fiber diameter produces a lower dust dislodgement efficiency. In Sample 6, the dust dislodgement efficiency improved by 1.3 times that of Sample 1, confirming that filters with lower fiber linear density have higher dust dislodgement efficiency. Filters with low fiber linear density have a large surface area of fibers; thus, dust easily accumulates on the fiber surface (i.e., on the surface of the felt) and is easily dislodged.

Furthermore, the second moment of the fiber area becomes smaller because of low fiber linear density; therefore, fibers are easily deformed by bending. Fibers easily fall forward with the pressure of dust collection and then form layers (Fig. 6). As a result, the clearance between fibers decreases, and it is difficult for dust to penetrate inside the felt; instead, it accumulates on the surface. Fibers are easily bent when dust is dislodged, and they deform along the flow channel. Therefore, the penetrating dust is carried by the fluid and then dislodged. We assume that dust accumulating on the surface is easier to dislodge when pulsed air is applied; therefore, dust dislodgement efficiency improves.

Dust accumulation inside the filter after the dust collection and dislodgment test is examined using a microscope (VW-6000, KEYENCE Co., Ltd.). Figure 7(a) presents a photo of the cross-section of Sample 1 filter, the reference product. Dust accumulates significantly on the surface of the filter to a depth of $1 / 4$ of the cloth thickness. Figure 7(b) is a photo of the cross-section of Sample 2 filter. The dust penetrates less than in Sample 1 and can be easily removed; thus, dust dislodgement efficiency is improved.

\section{Influence of fiber Young's modulus}

The tensile stress-strain curve of fibers were obtained using the tensile test machine described in foregoing section to investigate the influence of the mechanical characteristics of the fibers of bag filter felt on dust 
dislodgement efficiency. The initial inclination of the curve was defined as the modulus of fiber elasticity. The average modulus of elasticity was 1.84GPa for Sample 2, 2.86GPa for Sample 3, and 5.02GPa for Sample 5.

Figure 8 compares the dust dislodgement efficiencies that differ due to differences in modulus of elasticity of bag filter felt fibers. Samples 2, 3, and 5 have the same values of other parameters (e.g., diameter of fiber). The dust dislodgment efficiency of the filter decreases as the modulus of fiber elasticity increases.

As depicted in Fig. 6, the ease with which fibers fall on the surface of the felt due to the pressure of dust collection is related to the flexural rigidity of the fibers (the second moment of area $\times$ modulus of elasticity). Dust dislodgment efficiency is improved by the mechanism described in foregoing section.

Figure 9 depicts microscopic photos of the filter's surface after the dust collection and dislodgment test. More dust remains on the surface layer of Sample 3 (with a larger modulus of elasticity) than on that of Sample 2 (with a smaller modulus of elasticity). Thus, the residual pressure in the bag filter increases.

However, filters with a high modulus of elasticity of fibers have hard fibers in the felt and large clearances between fibers, due to the crossing of fibers. Therefore, the initial pressure loss is lower. During dust collection, dust easily penetrates the clearance between fibers inside the felt. When dislodging dust, fibers are hard and are difficult to deform along the flow channel, and thus hinder mine dust motion. Mine dust between fibers is no longer carried by fluid and dust dislodgment efficiency decreases. Figure 10 presents photos of the cross-section of the bag filter near the dust-collecting surface. Fibers in Sample 5 collapse less than in Sample 2.

\section{Influence of Fiber Cross-Section}

Figure 11 compares the dust dislodgment efficiencies of samples that have the same fiber linear density (2.2dtex) but different fiber cross-sections. Sample 1 has fibers with a circular solid cross-section, whereas Sample 4 has fibers with a triangular solid cross-section. Other sample parameters are the same. Sample 4 has higher dust dislodgment efficiency than Sample 1.

As depicted in Fig. 7, filters that consist of fibers with a triangular solid cross-section have a greater surface area than those that consist of fibers with a round cross-section with the same total weight, density, and fiber linear density. Dust easily accumulates on the surface of fibers, stays on the surface of the felt, and is easier to dislodge. We assume that dust dislodgment efficiency is improved for this reason. That is to say, the manner of dust sticking differs between fibers with different cross-sections, and therefore greatly influences dust dislodgment efficiency.

The fiber aggregate in bag filter felt greatly influences the dust dislodgement performance of dust collectors. To improve dust dislodgement performance, it is important that dust in gas does not penetrate the felt during dust collection but stays on its surface (dust-collecting surface) and accumulates there. It is also important that a channel through which pulsed jet fluid can flow easily is formed in the fiber aggregate during dust dislodgement, so that penetrated dust can be discharged to the outside. This effect of felt fibers suggests its mechanism.

\section{CONCLUSION}

We prepared prototypes of several types of bag filter with different fiber characteristics and conducted dust-collecting and dislodging tests to investigate the influence of the fiber structure of the bag filter felt (diameter, form, and Young's modulus of fiber) on dust dislodgment efficiency to improve the dust dislodgment efficiency of bag-filter dust collectors used in garbage-incinerating facilities and blast furnaces. We also explored its mechanism.

When the densities and thicknesses of the foundation cloth and filter used for the bag filter are the same, dust dislodgment efficiency is related to the diameter of the felt fibers: the smaller the fiber diameter, the higher the dust dislodgment efficiency. Mechanical characteristics of the felt fibers also influence dust dislodgment efficiency. In filters with large Young's modulus of fibers, dust dislodgment efficiency declines. Furthermore, 
fibers with a triangular cross-section provide higher dust dislodgment efficiency than those with a circular cross-section.

We assume that the state of multi-phase flow that contains powder and the mechanical characteristics of materials comprehensively influence the dust dislodgment efficiency of bag-filter dust collectors. The results obtained in this study are close to the characteristics of the filters used at actual sites. Therefore, we prepared these filters and subjected them to tests on the assumption of immediate practical applicability. When the parameters of the felt fibers change greatly (e.g., to extremely thick or thin fiber), further investigation will be necessary.

In our next paper, we will report on the influence of felt fiber material, and the surface treatment and influence of the structure of the bag filter (e.g., fundamental cloth and density).

\section{Acknowledgements}

We thank Mr. Sadanobu Uyama of Nihon Spindle Manufacturing Co., Ltd., for his useful advice and cooperation.

This study was conducted with subsidies from the Grants for Excellent Graduate Schools, MEXT, Japan.

\section{REFERENCES}

1. Japan the Society of Powder Technology, Device and technology of the dust-collection, THE NIKKAN KOGYO SHIMBUN Ltd 2001, pp133-182.

2. Hindy K, Sievert J, Löffler F, Influence of cloth structure on operational characteristics of pulse-jet cleaned filter bags, Environment International 1987; 2: 175-181.

3. Simon X, Chazelet S, Experimental study of pulse-jet cleaning of bag filters supported by rigid rings, Powder Technology 2007; 172: 67-81

4. Richard D, Cass R and Hall R, Dust Dislodgement from Woven Fabrics Versus Filter Performance, Journal of the Air Pollution Control Association 1987; 28: 1, 47-49.

5. Ellenbecker M and Leith D, Dust removal from Non-Woven Fabrics-Cleaning Methods Need to be improved, Filtration and Separation 1981; 316 -320.

6. Dennis R, Wilder J and Harmon D, Predicting pressure loss for pulse-jet filters, J. Air Pollution Control Association 1981; 31: 987-992.

7. Bao L, Yu M, Kishma, T, Uyama S and Kemmocchi K, Improving Bag Cleaning Efficiency of a Bag Filter Dust Collector Using Multiple Jet Pulses, Transactions of the Japan Society of Mechanical Engineers, Series C 2011; 77: 179-186.

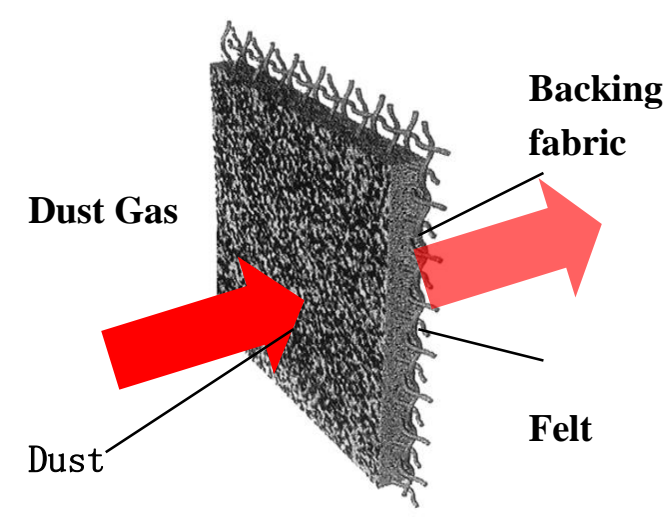


Fig. 1. Structure of a bag filter

(8)

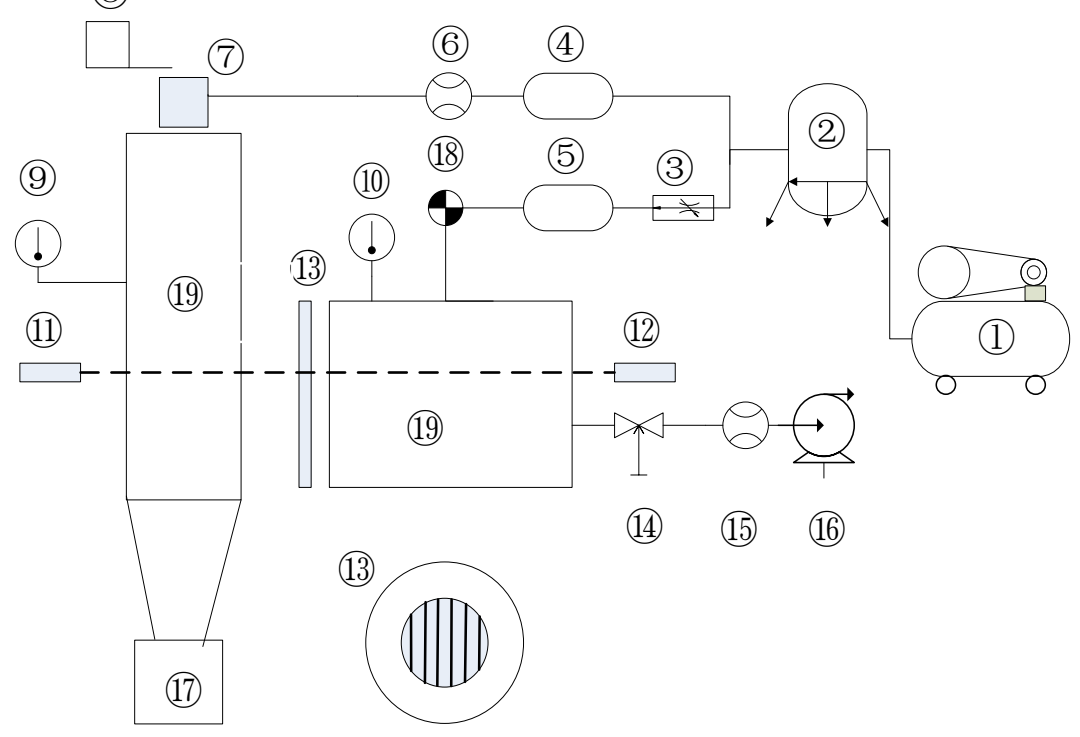
(1) Air compressor
(2)(4) (5) Air tank
(3) Regulator
(6)(15) Flow meter
(7) (8) Micro feeder
(9)(10) Manometer

(11) Laser displacement meter
(13) Bag filter
(14) Flow quantity control valve
(16) Blower
(17) Dust collection
(18) electromagnetic valve
(19) Gas diffusion space

Fig. 2 Diagram of test equipment for dust collection and dislodgement

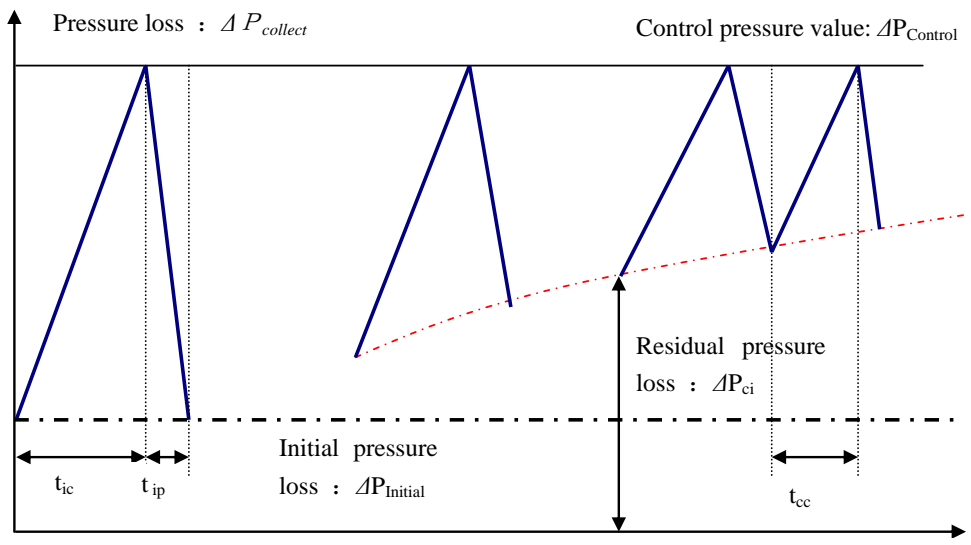

Dust collecting time : T

Fig.3 The relationship between pressure loss and time 


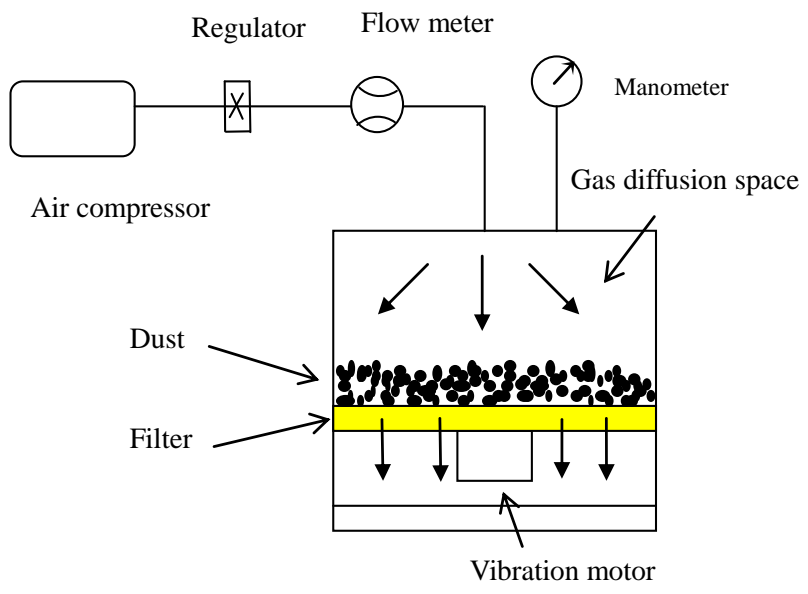

Fig.4 Schematic diagram of the shaking device

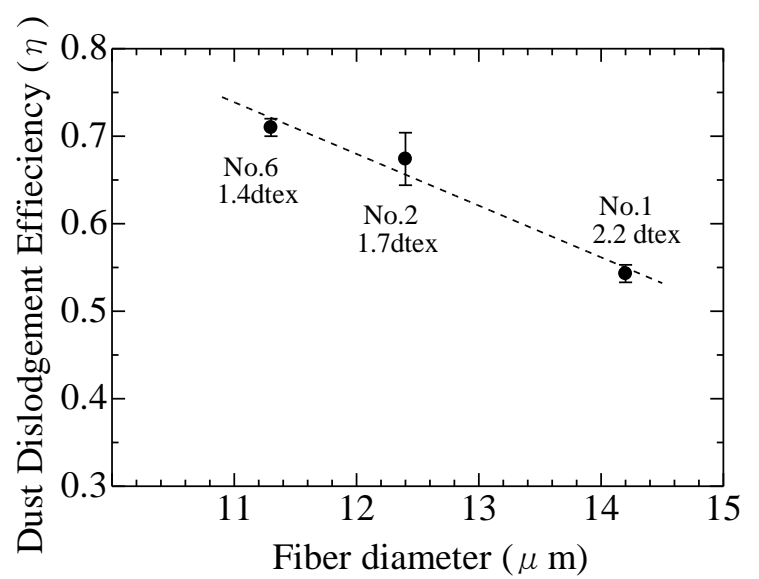

Fig.5 Dust dislodgement efficiency against fiber diameter among 3 samples

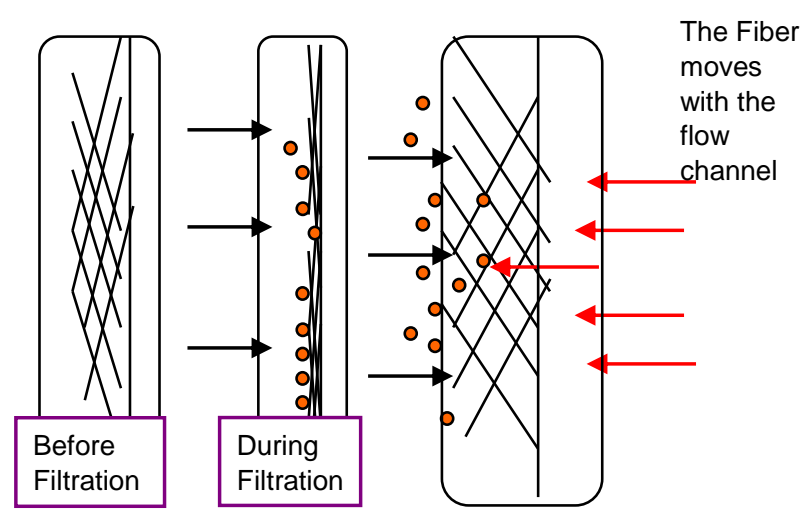

Fig.6 Schematic mechanism of dust attachment on the fiber. 


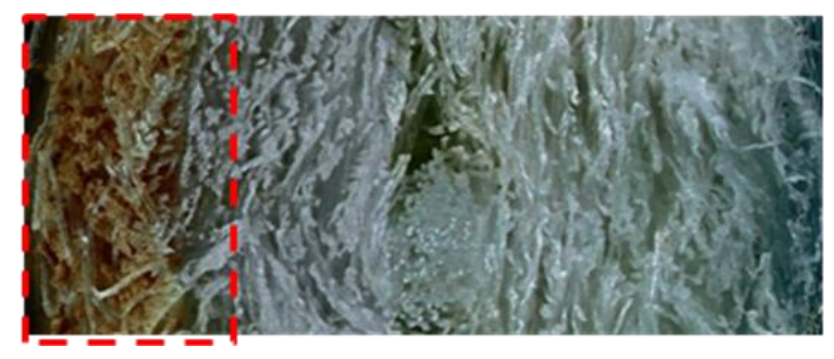

(a) Sample No.1

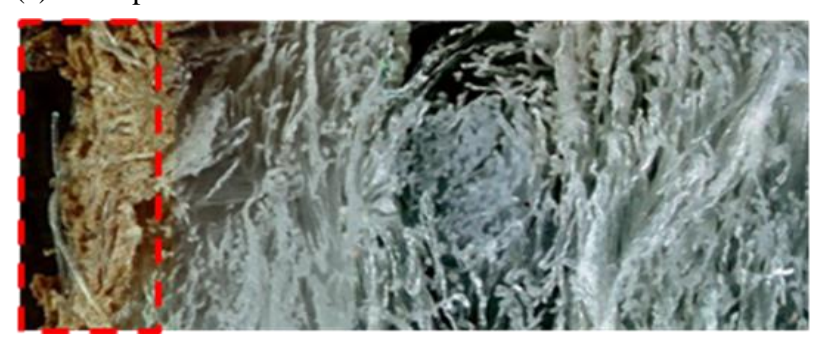

(b) Sample No.2

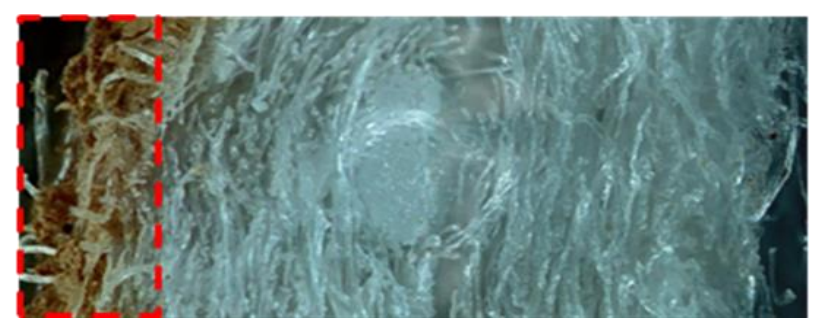

(c) Sample No.4

Fig.7 The section image of 3 test sample $(a, b, c)$ after filtration and dust dislodgement experiment.

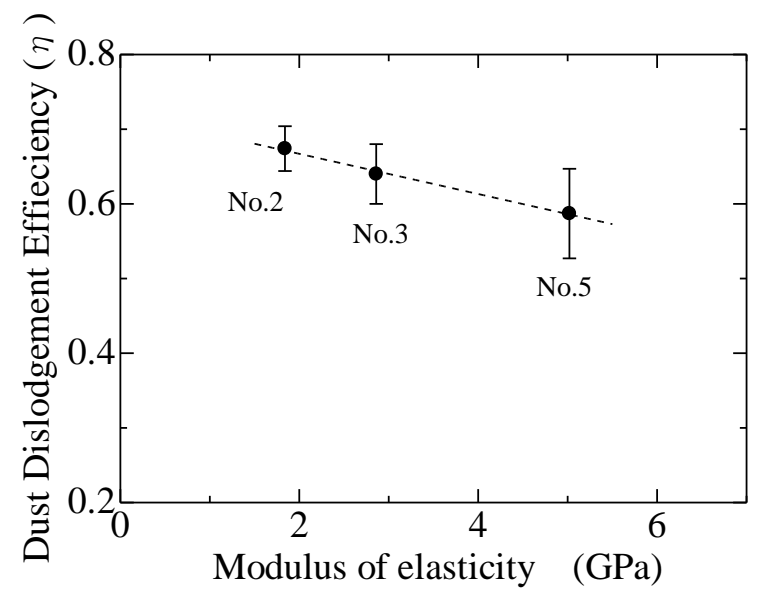

Fig.8 Dust dislodgement efficiency against fiber modulus of elasticity among 3 samples 


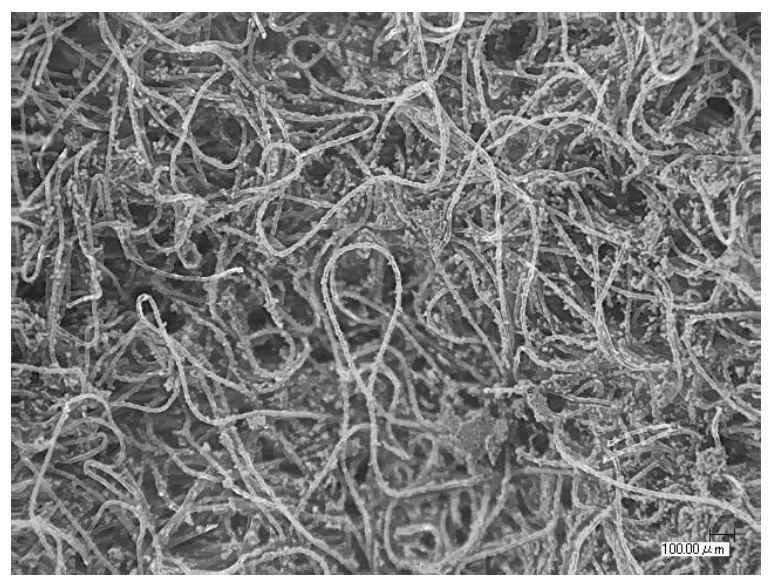

(a) Sample No.2

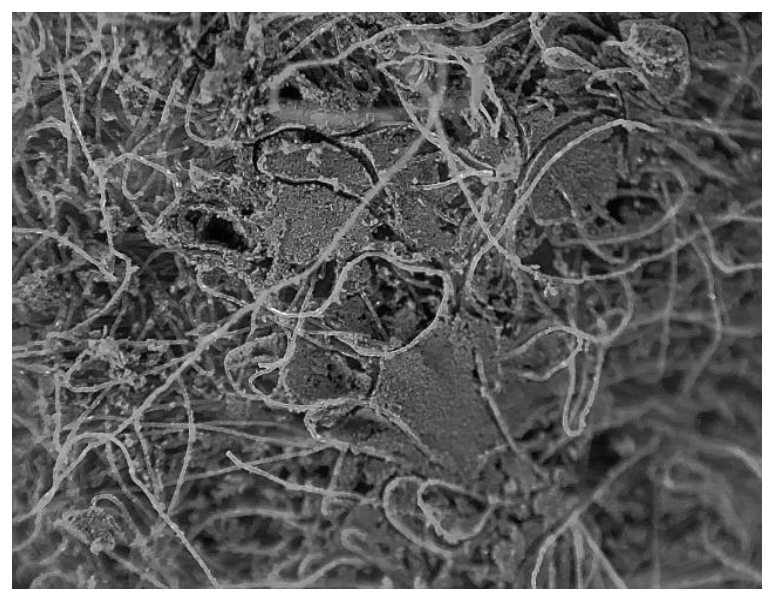

(b) Sample No.3

Fig.9 The surface image of 2 test samples (a,b) after filtration and dust dislodgement experiment

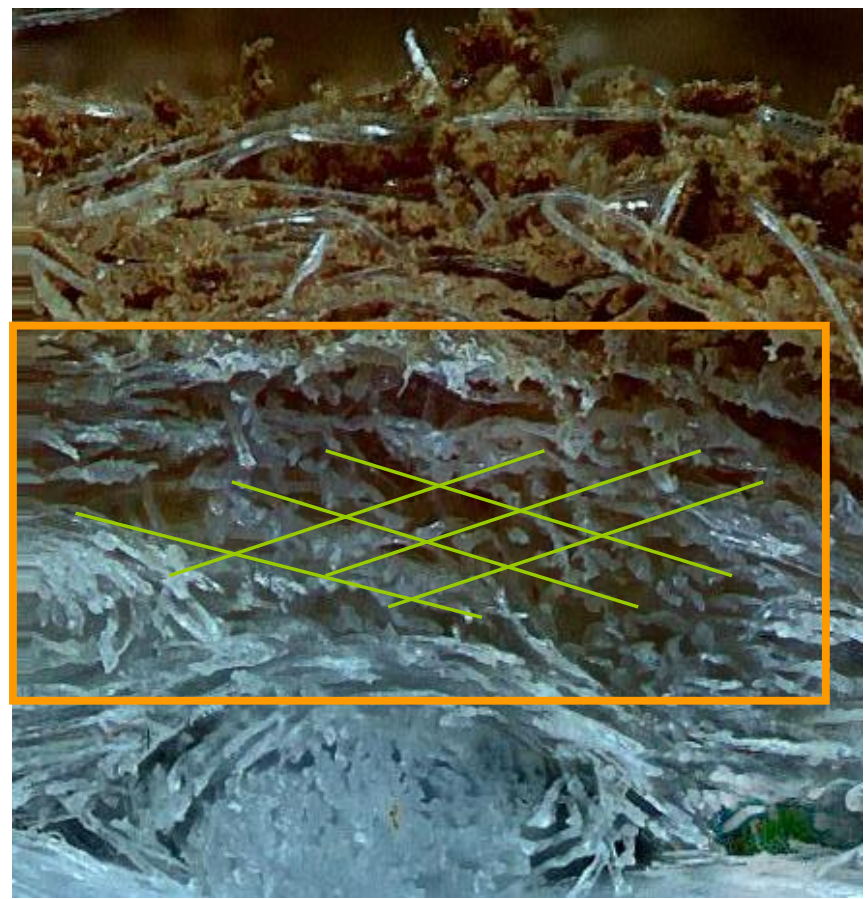

(a) Sample No2 


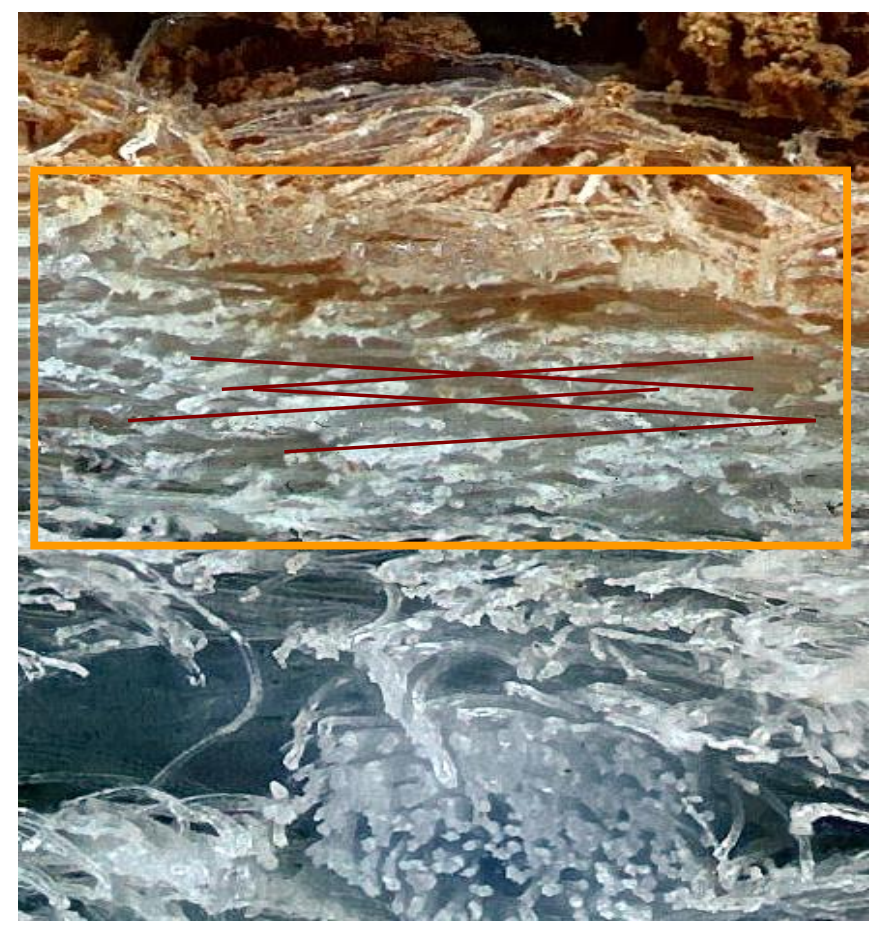

(b) Sample No.5

Fig.10 The section image of 2 test samples $(\mathrm{a}, \mathrm{b})$ after filtration and dust dislodgement experiment focusing on the its mechanism

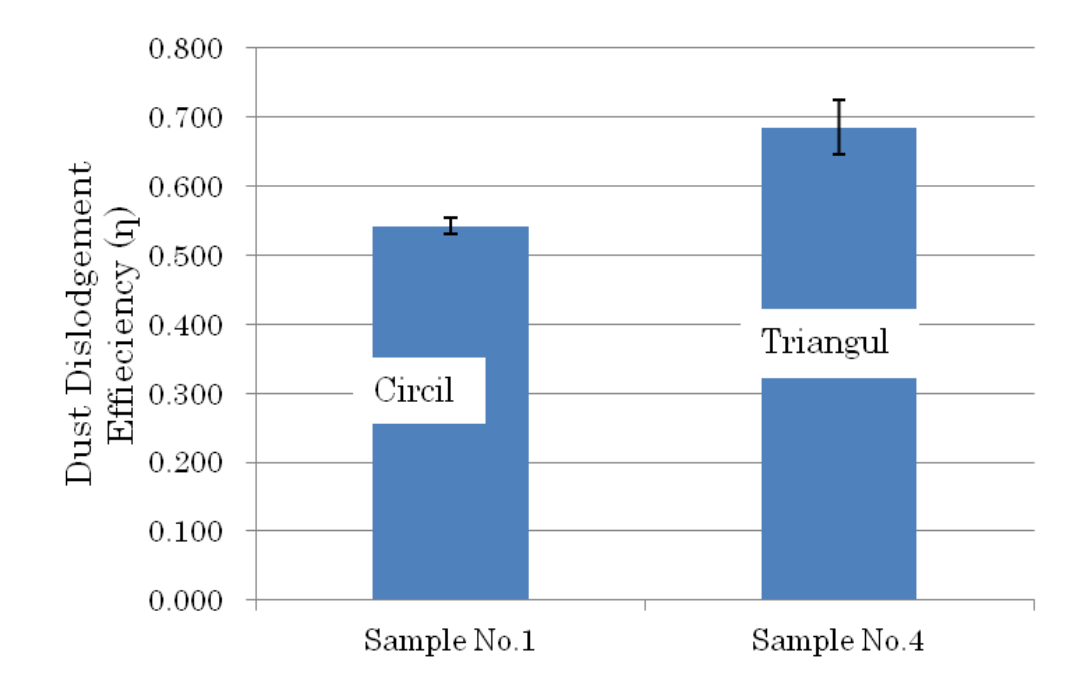

Fig. 11 Dust dislodgement efficiency against fiber cross-section

Table 1 Specification of Filter Samples

\begin{tabular}{|c|c|c|c|c|c|c|}
\hline No & Cross Section & $\begin{array}{c}\text { Fiber linear } \\
\text { density }[\mathrm{dtex}]\end{array}$ & $\begin{array}{c}\text { Fiber Strength } \\
{[\mathrm{cN} / \mathrm{dte}]}\end{array}$ & $\begin{array}{c}\text { Filter weight } \\
\text { per area }\left[\mathrm{g} / \mathrm{m}^{2}\right]\end{array}$ & $\begin{array}{c}\text { Filter density } \\
{\left[\mathrm{g} / \mathrm{m}^{3}\right]}\end{array}$ & $\begin{array}{c}\text { Filter thickness } \\
(\mathrm{mm})\end{array}$ \\
\hline 1 & Circular & 2.2 & 5.65 & 608 & 0.31 & 1.96 \\
\hline 2 & Circular & 1.7 & 4.85 & 613 & 0.31 & 1.97 \\
\hline & & & & & & \\
\hline
\end{tabular}




\begin{tabular}{|c|c|c|c|c|c|c|}
\hline 3 & Circular & 1.7 & 5.65 & 636 & 0.31 & 2.05 \\
\hline 4 & Triangular & 2.2 & 5.25 & 620 & 0.31 & 2.00 \\
\hline 5 & Circular & 1.7 & 6.62 & 583 & 0.29 & 2.01 \\
\hline 6 & Circular & 1.4 & 5.40 & 600 & 0.31 & 1.94 \\
\hline
\end{tabular}

Table 2 Dust collecting experiment conditions

\begin{tabular}{ll}
\hline Temperature & $20 \pm 5^{\circ} \mathrm{C}$ \\
\hline Humidity & $50 \pm 10 \%$ \\
Dust & JIS 8 type \\
Dust supply velocity & $0.02 \mathrm{~g} / \mathrm{s}$ \\
Dust Dispersion Velocity & $40 \mathrm{~L} / \mathrm{min}$ \\
Filtration velocity & $53 \mathrm{~L} / \mathrm{min}$ \\
\hline
\end{tabular}

Table 3 Dust dislodgement experiment conditions

\begin{tabular}{ll}
\hline Temperature & $20 \pm 5^{\circ} \mathrm{C}$ \\
\hline Humidity & $50 \pm 10 \%$ \\
Pulse pressure & $200 \mathrm{kPa}$ \\
Pulse duration & $0.05 \mathrm{~s}$ \\
Control pressure loss & $1000 \mathrm{~Pa}$ \\
\hline
\end{tabular}

